\title{
Studies of $\Upsilon$ decays at Belle
}

\author{
Mauro Verzetti* on behalf of Belle collaboration \\ Sezione INFN and Università di Torino, Dipartimento di Fisica Sperimentale, via Pietro Giuria \\ 1 - I-10125 Torino \\ E-mail: verzettidto.infn.it
}

In this paper we report some results obtained by the Belle collaboration exploiting samples taken at different energies. Analyzing a $21.7 \mathrm{fb}^{-1}$ data sample collected at the energy of the $\Upsilon(5 S)$ it was possible to observe an unexpected enhancement of the transitions $\Upsilon(5 S) \rightarrow \pi \pi \Upsilon(1,2,3 S)$ with respect to $\Upsilon(4 S)$. A scan sample around $\Upsilon(5 S)$ of $8.1 \mathrm{fb}^{-1}$ suggests the presence of a new resonance which wouldn't belong to the classical bottomonium framework. Using $23.6 \mathrm{fb}^{-1}$ at the energy of $\Upsilon(5 S)$ it was possible to quantify the fraction of the three body decay $\Upsilon(5 S) \rightarrow B^{(*)} \overline{B^{(*)}} \pi$. Finally, exploiting the $24 \mathrm{fb}^{-1}$ data sample taken at the energy of the $\Upsilon(2 S)$, corresponding to about 170 million $\Upsilon(2 S)$ decays, Belle claims the first observation of the rare E1 transition $\chi_{b 0} \rightarrow \gamma \Upsilon(1 S)$.

35th International Conference of High Energy Physics - ICHEP2010,

July 22-28, 2010

Paris France

${ }^{*}$ Speaker. 


\section{Introduction}

Belle collected samples at energies different from $\Upsilon(4 S)$ to perform QCD and BSM studies. In these proceedings, we summarize the results of three of these analyses. The first one is the first evidence of a peak in the production cross section in the $\pi^{+} \pi^{-} \Upsilon(1,2,3 S)$ channels at an energy slightly different from the nominal energy of $\Upsilon(5 S)$ [1]. The second analysis is the first evidence of a three-body decay of the $\Upsilon(5 S)$ [2]. In the last part we will show the preliminary results on the $\Upsilon(2 S)$ sample recorded by Belle, which is the world's largest.

\section{Dipion transitions to $\Upsilon(1,2,3 S)$ in the $\Upsilon(5 S)$ region}

The discovery of many charmonium-like vector mesons above open charm threshold that do not seem to easily fit into the conventional $c \bar{c}$ spectrum gave rise to different possible interpretations of this phenomenon like multiquark states, mesonic molecules or states with an excited gluonic degree of freedom (so called hybrids).

Using $21.7 \mathrm{fb}^{-1}$ of data taken on the $\Upsilon(5 S)$ peak in 2006 , Belle measured decay widths in $\Upsilon(1,2,3 S) \pi^{+} \pi^{-}$final state two orders of magnitude larger than the ones observed at the $\Upsilon(4 S)$ peak. In 2007 a six-point scan was performed in the proximity of the $\Upsilon(5 S)$ peak. The lineshape of the $\Upsilon(1,2,3 S) \pi^{+} \pi^{-}$cross sections were studied searching for the evidence of the $b \bar{b}$ analogue of $\mathrm{Y}(4260)[5]$.

A simultaneous $\chi^{2}$ fit of the three channels with a Breit-Wigner function (Fig. 1) gives a resonance mass of (10899.6 \pm 1.8$) \mathrm{MeV}$. The comparison with the most precise measurement of the mass of $\Upsilon(5 S)$, performed by BaBar fitting the shape of the resonance accounting for possible interferences between resonance and continuum [3], leads to a discrepancy of $3.4 \sigma$. The discrepancy with the PDG [6] nominal value is $5.1 \sigma$ instead.

This result stimulated new activity in the community of the theorists: some of these results are shown in this conference [4].

The last runs of the Belle era were devoted to perform a new scan of this region with 16 points $5 \mathrm{MeV}$ apart (50 $\mathrm{pb}^{-1}$ each point), an analysis exploiting this large statistic is expected.

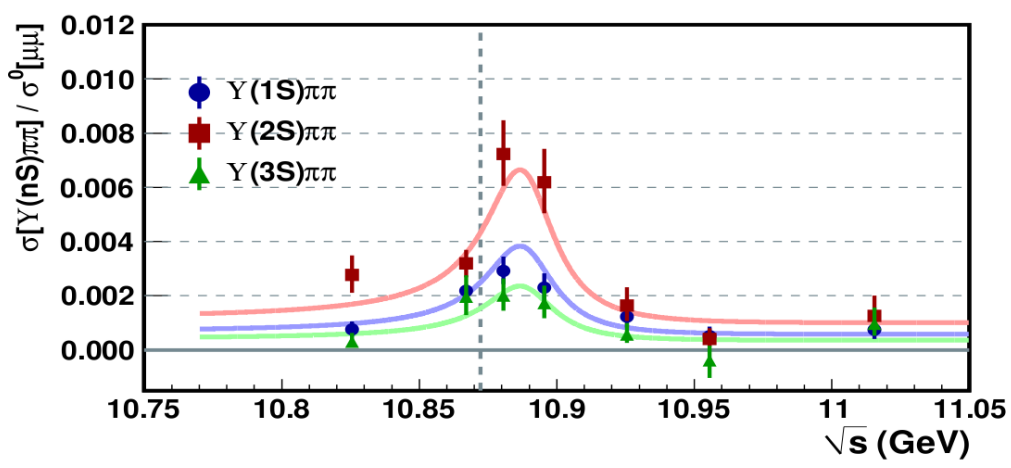

Figure 1: Simultaneous fit of the $\pi \pi \Upsilon(1,2,3 S)$ cross section in the scan region. The dashed line indicates the maximum of the hadronic cross section 


\section{Measurement of $\Upsilon(5 S)$ decays to $B^{0}$ and $B^{+}$mesons}

At the energy of the $\Upsilon(5 S), B$ mesons with different spin and flavour can be produced $\left(B, B^{*}\right.$, $\left.B_{s}, B_{s}^{*}\right)$. The phase space allows also the production of one or two extra pions together with a $B^{(*)}$ pair. The prompt decay of the $\Upsilon(5 S)$ in more than two bodies was never observed. In order to tag these events the $B^{ \pm}$'s were reconstructed in $J / \psi K^{ \pm}$and $D^{0} \pi^{ \pm}$(with $D^{0} \rightarrow K \pi$ and $K 3 \pi$ ) final states, while $B^{0}$ 's were reconstructed in the $J / \psi K^{* 0}$ and $D^{-} \pi^{+}$final states. The main source of background are the continuum events $\left(e^{+} e^{-} \rightarrow q \bar{q}\right.$ with $\left.q=u, d, s, c\right)$.

Plotting the distribution of the events in function of $\Delta E\left(=E_{B}^{*}-\sqrt{s} / 2\right)$ and $M_{\mathrm{bc}}$

$\left(=\sqrt{(\sqrt{s} / 2)^{2}-P_{B}^{*^{2}}}\right)$ it is possible to separate the different kind of decay. Fig. 2 shows the scatter plot of these two observables for MC events (a) or real data (b), in the $J / \psi \mathrm{K}$ decay channel. Projecting on $M_{\mathrm{bc}}$ the band shown in Fig. 2(b) it is clearly possible to distinguish (Fig. 3) three peaks due to the two body decays $\left(B \bar{B}, B \overline{B^{*}}, B^{*} \overline{B^{*}}\right)$, a broad bump due to the three body decays and a peak, most of which is due to ISR (i.e. radiative) production of a $\Upsilon(4 S)$.

In conclusion this analysis showed that $\left(17.5_{-1.6}^{+1.8} \pm 1.3\right) \%$ of the $b \bar{b}$ events at $\Upsilon(5 S)$ energy have more than two bodies in the final state (first measurement). The three body decay is dominated by $B \overline{B^{*}} \pi$ and $B^{*} \bar{B} \pi$, while in the region with $M_{\mathrm{bc}}>5.41 \mathrm{GeV} / \mathrm{c}^{2}$ the dominant phenomenon is ISR.

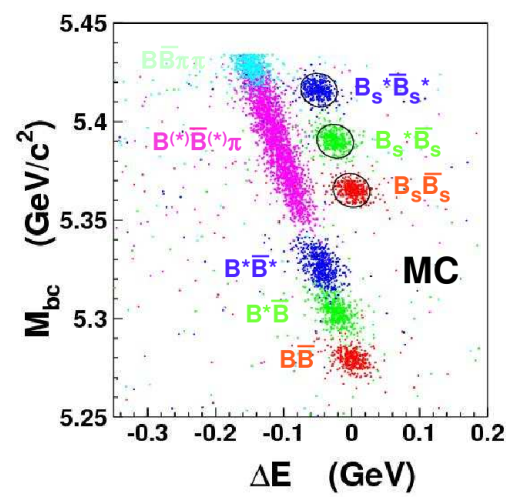

(a) $\mathrm{MC}$ events

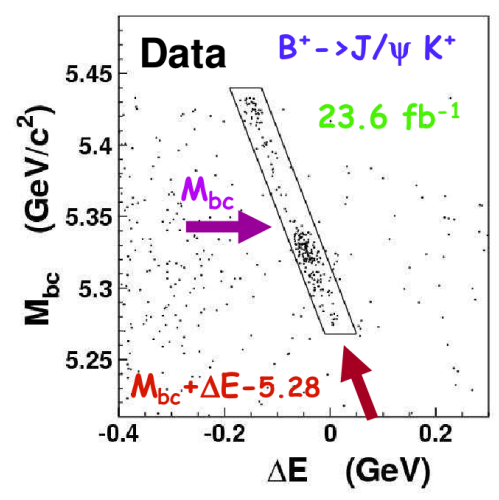

(b) Real data in $B^{+} \rightarrow J / \psi K^{+}$events

Figure 2: Scatter plot for MC and real data. $\Delta E\left(=E_{B}^{*}-\sqrt{s} / 2\right)$ vs. $M_{\mathrm{bc}}\left(=\sqrt{(\sqrt{s} / 2)^{2}-P_{B}^{*^{2}}}\right)$

\section{Observation of the radiative transition from $\chi_{b 0}$ to $\Upsilon(1 S)$}

The two data samples collected by Belle at the energy of the $\Upsilon(2 S)$ in $2008\left(6.5 \mathrm{fb}^{-1}\right)$ and 2009 $\left(17.6 \mathrm{fb}^{-1}\right)$ are the world largest $(\sim 171$ million decays). This enables us to look for the expected radiative E1 transition from $\chi_{b 0}$ to $\Upsilon(1 S)$. Double photon cascades between $\Upsilon(2 S)$ and $\Upsilon(1 S)$ have been observed to occur via $\chi_{b 1,2}$ intermediate states, since the early studies of bottomonium transitions. So far, the transition via $\chi_{b 0}$ has not yet been observed, as its branching ratio is expected to be an order of magnitude lower than the other two transitions.

The search was initially performed on a $\mu^{+} \mu^{-} \gamma \gamma$ sample for which a better purity of the signal can be achieved through (a) $\left|M_{\mu \mu}-M_{\Upsilon}(1 S)\right|<70 \mathrm{MeV}$, (b) $p_{\mu \mu}>250 \mathrm{MeV}$. 

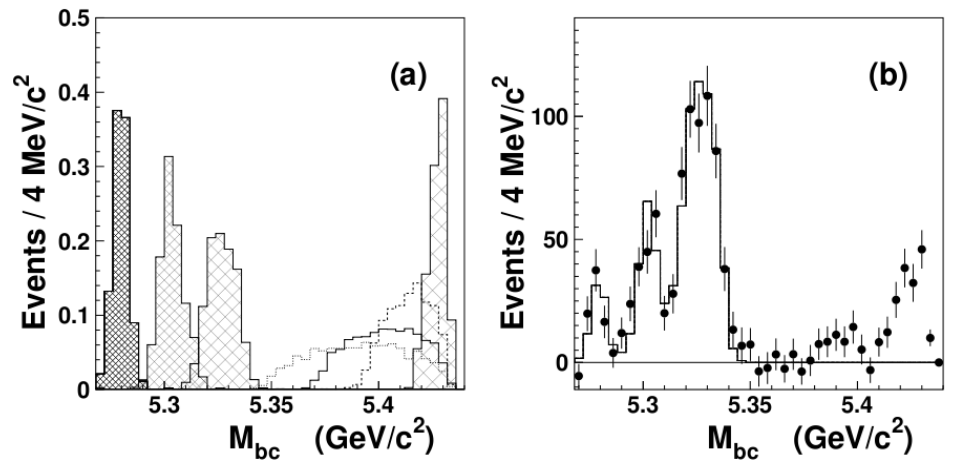

Figure 3: Projection of scatter plot on $M_{\mathrm{bc}}$ : (a) for MC data; (b) for real data

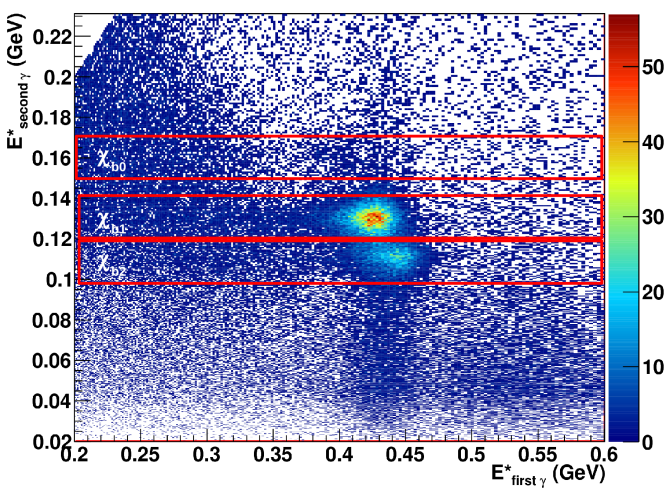

Figure 4: Energy of the most energetic photon vs. energy of the second one in $\mu \mu \gamma \gamma$ events

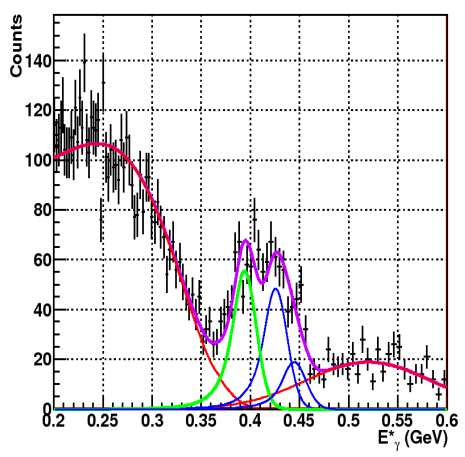

Figure 5: Projection of the $\chi_{b 0}$ band in exclusive events

Exploiting the better energy resolution at lower energies it is possible to separate the different $\chi_{b}$ transitions. In Fig. 4 the three bands show the regions where we expect the transitions to happen.

The PDF used to parametrize the signal, a Crystal Ball Function (CBF) with Doppler smearing, was tuned using the dominant $\chi_{b 1}$ peak. To take into account the effects of the asymmetry of the collider we divided our total acceptance in three bands in $\cos \theta^{*}$ (where $\theta^{*}$ is the azimuthal angle in the CM frame) and fitted them separately.

The energy band of the $\chi_{b 0}$ is contaminated by three main sources of background: the decay $\Upsilon(2 S) \rightarrow \pi^{0} \pi^{0} \Upsilon(1 S)$, the continuum radiative process $e^{+} e^{-} \rightarrow \mu^{+} \mu^{-} \gamma$, the $\chi_{b 1,2}$ cascades with one extra cluster. The first affects the low energy region and was parametrized using MC simulation, the second affects the high energetic region and can be easily described as a Gaussian fitted at energies higher than $480 \mathrm{MeV}$ while the third was described with a CBF with values averaged from the previous $\chi_{b 1}$ fits. The selection yielded a sample of $(481 \pm 28)$ events corresponding to a significance of about $17 \sigma$ (Fig. 5).

To look for the same signal in the inclusive single photon spectrum, we divided our hadronic sample in three bands in $\cos \theta^{*}$. We only looked at the energy region $[200,550] \mathrm{MeV}$ requiring 


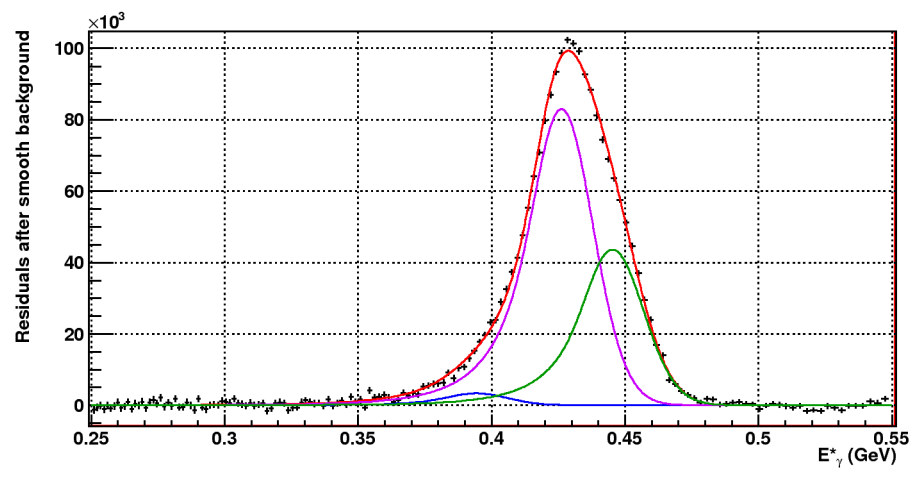

Figure 6: Description of the $\chi_{b}$ peaks in the inclusive single photon spectrum after the subtraction of the non-peaking (smooth) background

that the second Fox-Wolfram ratio of the event to be less than 0.98 and the absolute value of the cosine between the photon and the thrust axis of the remaining event to be less than 0.8 .

The spectrum was fitted using a $4^{\text {th }}$ degree polynomial, describing the non peaking background, summed to a linear combination of the appropriate PDF to describe the three $\chi_{b}$ peaks. This sample allows us to avoid the systematic introduced by the low energy photon efficiency and to measure the ratio $\chi_{b 0} \rightarrow \Upsilon(1 S) /\left(\chi_{b 1} \rightarrow \Upsilon(1 S)+\chi_{b 2} \rightarrow \Upsilon(1 S)\right)$. The yield of the $\chi_{b 0}$ signal is $57_{-8}^{+10} \times 10^{3}$ events, while the sum of the other two $\chi$ 's is $(1.960 \pm 0.013) \times 10^{6}$ events, therefore the ratio is $(2.9 \pm 0.5) \%$.

\section{References}

[1] K. F. Chen et al. [Belle Collaboration], Phys. Rev. Lett. 100 (2008) 112001 [arXiv:0710.2577 [hep-ex]].

[2] A. Drutskoy et al. [Belle Collaboration], Phys. Rev. D 81 (2010) 112003 [arXiv:1003.5885 [hep-ex]].

[3] B. Aubert et al. [BABAR Collaboration], Phys. Rev. Lett. 102 (2009) 012001 [arXiv:0809.4120 [hep-ex]].

[4] Ahmed Ali, these Proceedings.

[5] B. Aubert et al. [ BABAR Collaboration ], Phys. Rev. Lett. 95 (2005) 142001 [hep-ex/0506081].

[6] K. Nakamura et al. [ Particle Data Group ], J. Phys. G 37 (2010) 075021 